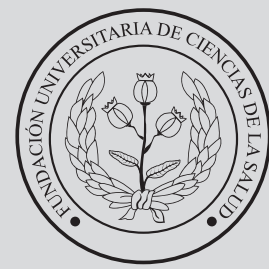

FUCS
Re 1

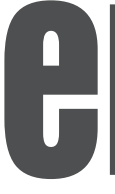

\section{de}

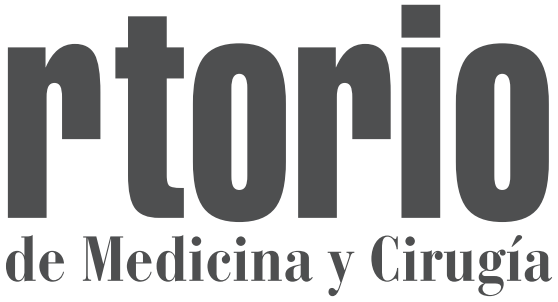

ISSN: 0121-7372 • ISSN electrónico: 2462-991X

Reporte de caso

\title{
Torsión aislada de trompa de falopio en niñas prepúberes
}

\section{Isolated torsion of the fallopian tube in premenarcheal girls}

${ }^{a}$ Facultad de Medicina, Universidad del Zulia; Servicio de Cirugía Pediátrica, Servicio Autónomo Hospital Universitario de Maracaibo, Venezuela.

\section{R ES U M EN}

La torsión aislada de la trompa de Falopio es una causa poco común de dolor abdominal agudo. Se describen dos casos clínicos de niñas prepúberes de 10 y 13 años de edad, con manifestaciones clínicas compatibles con apendicitis aguda, en quienes se evidenció torsión de la trompa derecha con signos de necrosis en la intervención quirúrgica. La existencia de esta entidad debe ser considerada en niñas con dolor abdominal agudo para evitar la lesión irreversible de la trompa.

Palabras clave: torsión; trompa; aislada; niñas prepuberales

(C) 2019 Fundación Universitaria de Ciencias de la Salud - FUCS. Este es un artículo Open Access bajo la licencia CC BY-NC-ND (http://creativecommons.org/licenses/by-nc-nd/4.0/).

\section{A B S T R A C T}

Isolated torsion of the fallopian tube is a rare cause of abdominal pain. We report on two premenarcheal girls, ages 10 and 13 years, who presented with clinical manifestations compatible with acute appendicitis, in whom, torsion of the right tube with signs of necrosis was evidenced during surgical intervention. This condition should be considered in all girls with acute abdominal pain to avoid irreversible damage to the tube.

Key words: torsion; tube; isolated; premenarcheal girls

(C) 2019 Fundación Universitaria de Ciencias de la Salud - FUCS. This is an open access article under the CC BY-NC-ND license (http://creativecommons.org/licenses/by-nc-nd/4.0/).

\section{INFORMACIÓN DEL ARTÍCULO}

Historia del artículo:

Fecha recibido: mayo 3 de 2018

Fecha aceptado: marzo 18 de 2019
Autor para correspondencia.

Dra. Grisel Maria Marín selmarin@yahoo.com
DOI

10.31260/RepertMedCir.v28.n3.2019.963 
La torsión aislada de las trompas de Falopio es una causa poco común de dolor abdominal agudo. ${ }^{1}$ Es un evento que rara vez se reporta, tiene una incidencia de 1 en 1.500 .000 mujeres en edad reproductiva y es menos frecuente en los grupos de edad pediátrica y adolescente. ${ }^{2,3}$ Fue descrita por BlandSutton en $1890 .{ }^{4}$ La torsión alrededor del pedículo vascular conlleva a la obstrucción del retorno venoso, que causa rápida congestión, isquemia y necrosis, con consecuencias futuras en la capacidad reproductiva. ${ }^{5}$ Esta condición no suele diagnosticarse en el preoperatorio ${ }^{6,7}$ debido a que los síntomas son inespecíficos y comunes con otras patologías, ${ }^{8}$ de manera que es en el momento en que se lleva a cabo la cirugía cuando se detecta el anexo comprometido. ${ }^{6,7}$ El diagnóstico temprano y la intervención quirúrgica son fundamentales para mantener la viabilidad de la trompa y preservar la fertilidad futura. ${ }^{2,9} \mathrm{En}$ este trabajo presentamos dos casos de dolor abdominal agudo en adolescentes prepúberes causado por torsión aislada de la trompa de Falopio.

\section{Caso 1}

Adolescente de 13 años de edad que no ha presentado menarquía, quien consulta por dolor de intensidad moderada a fuerte en fosa iliaca derecha de 8 horas de duración, acompañado de 2 episodios de vómito. Al examen físico se encontraba en buenas condiciones, temperatura corporal de $37^{\circ} \mathrm{C}$ y frecuencia cardiaca de 90 latidos por minuto. El abdomen presentaba signos de irritación peritoneal localizados en fosa iliaca derecha. Los exámenes de laboratorio reportaron leucocitos $6.950 \mathrm{x} \mathrm{mm}^{3}$ con $82 \%$ neutrófilos, hemoglobina $12,5 \mathrm{~g} / \mathrm{dL}$ y hematocrito $40,4 \%$. Uroanálisis normal. El ecograma abdominal reportó gran dilatación de asas colónicas aperistálticas en fosa iliaca derecha (figura1). Ingresó con diagnóstico de apendicitis aguda. La intervención quirúrgica a través de incisión transversa en fosa iliaca derecha evidenció abundante líquido serohemático en cavidad abdominal, trompa de Falopio derecha torcida sobre su eje, necrosada, con contenido hemorrágico. Apéndice cecal, ovario derecho, útero y anexos izquierdos de aspecto normal. Se realizó salpingectomía derecha con preservación del ovario. El resultado del estudio de anatomía patológica demostró necrosis de la trompa, hematosálpinx con endosalpingiosis.

\section{Caso 2}

Escolar femenina de 10 años de edad, quien consultó por presentar dolor abdominal de moderada a fuerte intensidad en fosa iliaca derecha de 12 horas de evolución, acompañado de vómito y fiebre. Al examen físico se encontró en regulares condiciones, deshidratación leve, temperatura $38^{\circ} \mathrm{C}$ y frecuencia cardiaca de 110 latidos por minuto. El abdomen era blando, doloroso a la palpación en fosa iliaca derecha, con signos localizados de irritación peritoneal. El laboratorio reportó leucocitos 12.840 x mm3 con $70 \%$ de neutrófilos. Ingresó con diagnóstico de apendicitis aguda. Durante la cirugía se evidenció trompa derecha torcida sobre su eje, necrosada y muy dilatada con líquido en su interior. Ovario derecho normal (figura 2). La biopsia reveló hidrosálpinx, congestión y hemorragia de la pared.

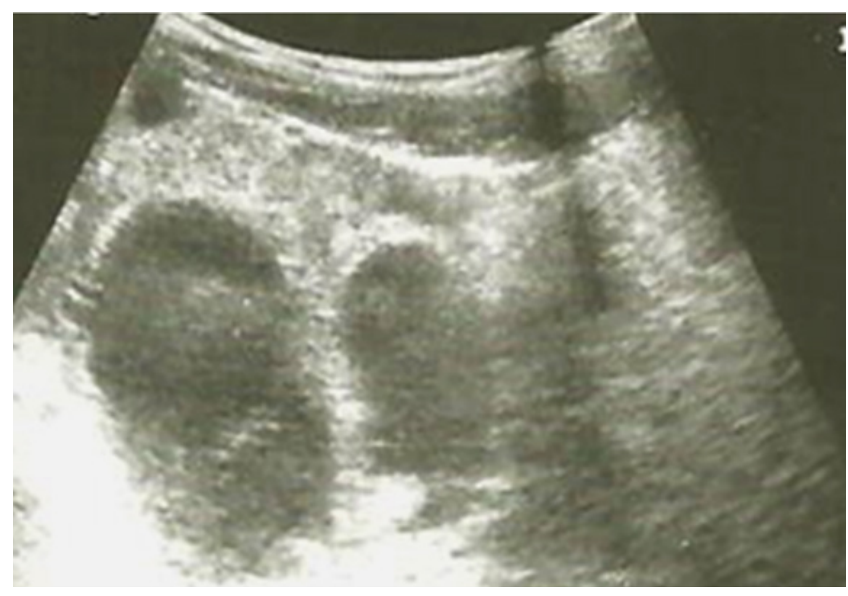

Figura 1. Econograma abdominal. Dilataciones quísticas en fosa ilíaca derecha que semejan asas intestinales dilatadas.

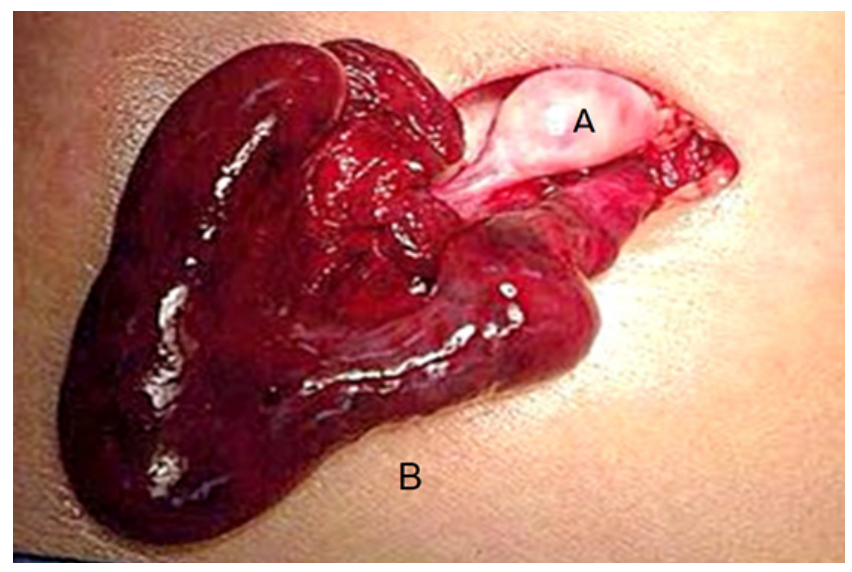

Figura 2. A. Ovario derecho de aspecto y configuración normal. B. Trompa de Falopio derecha torcida con hidrosálpinx.

\section{DIS CUSIÓN}

El diagnóstico de torsión de la trompa de Falopio en mujeres adolescentes a menudo no es clínico, sino que se realiza durante la laparotomía o laparoscopia. Se debe en parte a que las características clínicas de esta patología no están bien establecidas debido a su relativa rareza. Además, a pesar de la naturaleza aguda de la torsión, las manifestaciones abdominales son poco frecuentes o pueden ser inespecíficas. La apendicitis aguda es, por lo tanto, el diagnóstico erróneo más frecuente en las adolescentes que presentan torsión de trompa, como lo fue en los casos aquí presentados. ${ }^{5}$ 
Hidrosálpinx es una palabra griega que significa trompa de Falopio llena de agua o fluido, debido a una obstrucción distal completa con distensión anormal de la porción ampular. Aunque los cilios del revestimiento interno (endosálpinx) pulsan hacia el útero, el fluido tubárico se descarga por lo regular hacia el final de las fimbrias en la cavidad peritoneal donde se absorbe. Si la obstrucción de las fimbrias impide el libre paso de fluido a través de la trompa, se acumula y cambia de dirección hacia el útero, la trompa se distiende distalmente, se llena de líquido y no puede participar en el proceso reproductivo. ${ }^{10}$

Boukaidi y col. publicaron la serie más larga descrita de torsión aislada de trompa de Falopio asociada con hidrosálpinx en niñas y adolescentes sexualmente inactivas. Ellos encontraron ambas trompas dilatadas y con líquido en su interior (hidrosálpinx).

La torsión aislada suele manifestarse con aparición abrupta de dolor en hemiabdomen inferior, ${ }^{8,10-12}$ el cual se describe como constante o intermitente ${ }^{2}$ en el lado afectado, pero puede irradiarse hacia el flanco o el muslo. ${ }^{1,7,13}$ Hay síntomas asociados como náusea, vómito, anorexia, fiebre, sangrado vaginal y anormalidades intestinales o vesicales que pueden estar o no presentes. ${ }^{2}$ Los estudios de laboratorio por lo general se encuentran dentro de límites normales, ${ }^{11}$ pero pueden aparecer leucocitosis leve y elevación de la proteína $\mathrm{C}$ reactiva o de la velocidad de sedimentación globular. ${ }^{9}$ El examen abdominal puede mostrar dolor a la descompresión, defensa muscular o una masa anexial ${ }^{13}$ que también puede palparse durante el examen vaginal. ${ }^{7} \mathrm{El}$ paciente se encuentra hemodinámicamente estable y los signos de peritonitis se manifiestan con la necrosis de la trompa. ${ }^{14}$ La causa de la torsión tubárica es desconocida, en especial cuando no se asocia con torsión del ovario. ${ }^{5,11}$ Puede ocurrir tanto en trompas sanas como en afectadas por alguna dolencia. Los factores predisponentes pueden clasificarse en intrínsecos y extrínsecos. Entre los primeros están las alteraciones congénitas de la trompa como un recorrido tortuoso o una excesiva longitud, o bien adquiridas como hidrosálpinx, hematosálpinx, neoplasias, cirugía previa y una peristalsis anormal. Entre los extrínsecos se encuentran las alteraciones de los órganos vecinos (neoplasias, adherencias), movimientos o traumatismos de órganos pélvicos y el embarazo. ${ }^{1,2,7-9,12,13}$

En la edad adulta las principales causas de hidrosálpinx son la enfermedad inflamatoria pélvica, embarazo ectópico, endometriosis, cirugías abdominales previas y el antecedente de peritonitis o tuberculosis. Ningún dato similar está disponible en la población pediátrica. En la serie de Boukaidi y col. ninguna de las pacientes tenía antecedente médico sugestivo de enfermedad inflamatoria pélvica, apendicitis o endometriosis. Todas eran adolescentes sexualmente inactivas y negaban cirugía abdominal previa. Una posible etiología de torsión aislada asociada con hidrosálpinx, podría por lo tanto ser una malformación congénita de la trompa que se revela en el período peripuberal.

Entre 9 y 14 años el eje reproductivo cursa con altos niveles de FSH antes del inicio de la menstruación, lo cual puede activar la función ovárica y tubárica y revelar una oclusión distal asintomática de la trompa. Un episodio de inflamación pélvica asintomática durante la infancia, localizada cerca de las trompas, podría causar obstrucción distal, hidrosálpinx y torsión. La torsión de una hidátide de Morgagni localizada cerca del extremo fimbriado de las trompas, puede llevar a un diagnóstico erróneo y ser en realidad la causa del proceso patológico. ${ }^{10}$

La torsión es más común en el lado derecho con una tasa de 3:2 a 2:1 y por lo regular ocurre durante la ovulación. ${ }^{5}$ Esto podría explicarse por el hecho de que la movilidad de la trompa izquierda está limitada por el colon sigmoide o debido al flujo venoso lento del lado derecho, lo que puede provocar congestión. El otro motivo es que se operan más casos de dolor del lado derecho por sospecha de apendicitis aguda, mientras que los casos del lado izquierdo pueden resolverse de manera espontánea. Rara vez ocurre antes de la menarquía o durante la menopausia. Esta dispersión de frecuencias se debe aparentemente a que la mayoría de los factores de riesgo para la torsión ocurren en el grupo de edad reproductiva. $\mathrm{Su}$ similitud con otros procesos abdominales agudos suele dificultar y demorar el diagnóstico, lo que puede afectar en consecuencia la capacidad reproductiva de la mujer. ${ }^{12}$ El ultrasonido es a menudo la primera modalidad de examen imagenológico en una mujer con dolor abdominal o pélvico agudo, ya que no es invasivo ni hay exposición a la radiación. ${ }^{8}$ También la tomografía computarizada y la resonancia magnética se consideran útiles para detectar anomalías del ovario o la trompa. La apariencia ecográfica de una trompa torcida es variable e incluye una masa quística que a veces se extiende hacia la línea media, otras veces es compleja con líquido libre o bien se ve una estructura tubular o afilada que contiene desechos, fluido o hemorragia, con paredes engrosadas y pliegues mucosos protuberantes junto con el ovario ipsilateral normal. Puede ser difícil diferenciar entre masas ováricas, hidrosálpinx no complicado y torsión aislada de la trompa. En ocasiones el útero se encuentra desviado hacia el lado afectado. ${ }^{9}$ Se describe un signo sonográfico de torbellino después del movimiento de balanceo del transductor sobre la masa, que podría ser específico para la torsión de la trompa de Falopio. ${ }^{2,9}$ El doppler color puede ser de gran utilidad ya sea mostrando ausencia de flujo o uno de alta impedancia. Sin embargo, la presencia de flujo arterial y venoso normales no excluye la torsión debido a la doble vascularización del ovario y las trompas. ${ }^{2,9,10}$ La tomografía computarizada o la resonancia magnética ayudan a determinar el contenido de la estructura y pueden mostrar una trompa enrollada o un nudo de torsión. Además, puede revelar infarto mediante la falta de realce de contraste de las paredes tubáricas. Los hallazgos secundarios incluyen líquido intrapélvico libre, tejido adiposo peritubular, engrandecimiento y engrosamiento del ligamento ancho e íleo regional. ${ }^{9}$ Según Masafumi Toyoshima y col. ${ }^{8}$ la tomografía computarizada es muy útil para excluir la apendicitis y las imágenes reformadas multiplanares de los estudios tomográficos permiten visualizar hallazgos típicos, como una estructura dilatada llena de líquido, extremos cónicos y la configuración 
de la masa en la torsión. Aunque la resonancia no se usa de rutina en urgencias, el contraste superior de los tejidos blandos y la capacidad multiplanar permiten distinguir el ovario normal de una masa quística. Estos estudios permiten realizar el diagnóstico preoperatorio correcto de torsión aislada de trompa de Falopio debido a la presentación clínica combinada con las imágenes. Sin embargo, según Matthew y col. ${ }^{2}$ el valor de estas modalidades de imágenes sigue siendo controversial y puede dar lugar a un retraso innecesario de la intervención quirúrgica. La sensibilidad diagnóstica del ultrasonido, la tomografía computarizada, y la resonancia magnética ha sido reportada en un $22 \%, 14 \%$ y $40 \%$ respectivamente. La laparoscopia es considerada la "regla de oro" del diagnóstico y tratamiento, y es obligatoria cuando se sospecha torsión. ${ }^{3}$ Los diagnósticos diferenciales incluyen tanto patologías ginecológicas como no ginecológicas. Las primeras son torsión o ruptura de un quiste de ovario, enfermedad inflamatoria pélvica, embarazo ectópico y ruptura de un quiste del cuerpo lúteo. Las no ginecológicas incluyen apendicitis, cistitis, cólico biliar o renal, perforación de una víscera hueca, obstrucción intestinal, adenitis mesentérica y hernias estranguladas. ${ }^{6} \mathrm{La}$ apendicitis aguda y la torsión del ovario, representan los dos errores diagnósticos más frecuentes asociados con la torsión de la trompa. ${ }^{11}$

El abordaje quirúrgico depende de los datos preoperatorios de la edad y los deseos reproductivos. ${ }^{5}$ El tratamiento quirúrgico conservador para maximizar la preservación de la fertilidad debe ser el objetivo del tratamiento en mujeres jóvenes nulíparas y en embarazadas. La conservación de trompas potencialmente funcionales, una vez descartado el cáncer, siempre debe intentarse. El éxito depende de la duración de la torsión y el grado de daño tisular en el momento de la intervención; la cirugía temprana favorece la conservación de la trompa y la preservación de la fertilidad, aunque no se ha descrito la correlación entre la duración de los síntomas clínicos y la tasa de salpingectomía. Esta conservación es poco usual debido a la dificultad de realizar el diagnóstico preoperatorio correcto. ${ }^{8}$ Los procedimientos disponibles incluyen la resección tradicional cuando la trompa no puede recuperarse, como se realizó es estos dos casos, o la fijación tubárica si la torsión es incompleta y/o los tejidos permanecen viables, en especial en mujeres jóvenes para preservar la fertilidad..$^{5,10}$ que pueden realizarse mediante laparotomía o laparoscopia, dependiendo de las posibilidades técnicas. ${ }^{5}$ La diferenciación entre la necrosis y el estrangulamiento de la trompa torcida con viabilidad potencial es desafiante y existe un riesgo teórico adicional de embolia pulmonar si se intenta destorcer después de ocurrida la trombosis. La resección del ovario no está indicada si solo la trompa está involucrada en la torsión. ${ }^{5}$ Boukaidi y col. ${ }^{10}$ propusieron el manejo quirúrgico conservador de la torsión tubárica aislada asociada con hidrosálpinx en niñas y adolescentes en 2 pasos. Primero: tratamiento del episodio agudo de torsión, ${ }^{1}$ exploración laparoscópica de la pelvis y corrección cuidadosa de la torsión, ${ }^{2}$ punción y evacuación del hidrosálpin $\mathrm{x}^{3}$ y análisis citológico y bacteriológico del líquido (para descartar malignidad y salpingitis). Con el fin de no operar con la trompa edematosa e isquémica, ellos creen que es mejor opción una segunda cirugía programada pocas semanas después de la torsión. Segundo paso: salpingoscopia y salpingectomía o salpingoneostomía dependiendo del estado de la trompa. Debido a que el período de recurrencia promedio observado en sus casos fue de 68 días (43-83 días), ellos creen que 3 semanas después de la torsión sería un intervalo razonable para la reintervención.

Como se sabe, cuando hay compromiso del ovario en la población pediátrica, la corrección de la torsión y salvación del ovario es una opción segura, sin importar qué tan necrótico parezca el ovario. La misma filosofía podría aplicarse para la trompa en la torsión aislada asociada con hidrosálpinx después de una evaluación preliminar mediante salpingoscopia durante un segundo procedimiento quirúrgico (second look), para preservar sólo las trompas potencialmente funcionales. ${ }^{10} \mathrm{~A}$ las adolescentes con torsión tubárica se les debe realizar un seguimiento después del tratamiento, recomendando buscar atención médica de inmediato si experimentan síntomas similares en el futuro, ya que existe la posibilidad de torsión y necrosis del anexo contralateral lo que produciría un desastroso efecto emocional y reproductivo. ${ }^{5}$

\section{CONCLUSIÓN}

La torsión aislada de trompa de Falopio debe considerarse entre los diagnósticos diferenciales de dolor abdominal agudo en la población pediátrica, ya que el fracaso o el retardo en el diagnóstico correcto pueden conllevar a necrosis y daño irreversible de la trompa con repercusiones importantes en la capacidad reproductiva futura.

\section{CONFLICTO DE INTERÉS}

Los autores declaramos no tener ningún conflicto de interés.

\section{REFERENCIAS}

1. Bakshi R, Mehta A. Isolated Torsion Of The Fallopion Tube With Hydrosalpinx: A Rare Presentation. The Internet Journal of Gynecology and Obstetrics. 2008; 10(2): 1-3.

2. Blitz MJ, Appelbaum H. Management of Isolated Tubal Torsion in a Premenarchal Adolescent Female with Prior Oophoropexy: A Case Report and Review of the Literature. J Pediatr Adolesc Gynecol. 2013; 26(4): e95-e97. doi: 1016/j.jpag.2013.02.002 
3. Stemmer SM, Shurshalina A. Isolated Fallopian Tube Torsion: Detorsion and Tubal Preservation. CRSLS. e2015.00022. doi: 10.4293/CRSLS.2015.00022.

4. Bland J. Salpingitis and some of its effects. Lancet. 1890; 136(3509):1146-8. doi: 10.1016/S0140-6736(02)19799-6

5. Rizk D, Lakshminarasimha B, Joshi S. Torsion of the Fallopian Tube in an Adolescent Female: A Case Report. J Pediatr Adolesc Gynecol 2002; 15(3): 159-161. doi: 10.1016/S1083-3188(02)001493

6. Bharathi A, Gowri M. Torsion of the Fallopian Tube and the Haematosalpinx in Perimenopausal Women - A Case Report. J Clin Diagn Res. 2013; 7(4): 731-33.

7. Jalaguier-Coudray A, Thomassin I, Chereau E, Rouzier R, Darai E, Bazot M. A case or isolated torsion of the fallopian tube: Added value of magnetic resonance imaging. European Journal of Radiology Extra. 2011; 79(1): el-e4. doi: 10.1016/j. ejrex.2011.03.007

8. Toyoshima M, Mori H, Kudo K, Yodogawa Y, Sato K, Kudo T et al. Isolated torsion of the fallopian tube in a menopausal woman and a pre-pubertal girl: two case reports. J Med Case Rep. 2015; 9: 258-63. doi: 10.1186/s13256-015-0745-y
9. Van der Zanden M, Nap A, van Kints M. Isolated torsion of the fallopian tube: a case report and review of the literature. Eur J Peditr. 2011; 170(10): 1329-32. doi: 10.1007/s00431-011-1484-8

10. Boukaidi SA, Delotte J, Steyaert H, Valla JS, Sattonet C, Bouaziz $\mathrm{J}$ et al. Thirteen cases of isolated tubal torsions associated with hydrosalpinx in children and adolescents, proposal for conservative management: retrospective review and literature survey. J Pediatr Surg 2011; 46(7): 1425-31.

11. Daponte A, Dimopoulos KD, Terzis A, Messinis IE. Isolated fallopian tube torsion: a rare diagnosis in a perimenopausal patient with adnexal mass and raised Ca 19-9. Endoscopic management and review. Gynecol Surg. 2005; 2: 283-86.

12. García C, Parra F. Torsión aislada de trompa de Falopio: a propósito de un caso. Radiología. 2011; 53(1): 86-9.

13. Goktolga U, Ceyhan T, Ozturk H, Gungor S, Zeybek N, Keskin U et al. Isolated torsion of fallopian tube in a premenarcheal 12-yearold girl. J. Obstet. Gynaecol. Res. 2007; 33(2): 215-7.

14. Costa JR, Moohan J. Torsion of a fallopian tube: a rare cause of lower abdominal pain in a female of reproductive age. Sri Lanka Journal of Obstetrics and Gynaecology. 2011; 33: 63-4. 